\title{
An alternative cannulation approach for venovenous extracorporeal membrane oxygenation in children for long-term ambulatory support
} \author{
Stanford, Calif
}

Katsuhide Maeda, MD, PhD, ${ }^{a}$ Kathleen Ryan, MD, ${ }^{b}$ Carol K. Conrad, MD, ${ }^{c}$ and Vamsi V. Yarlagadda, MD,

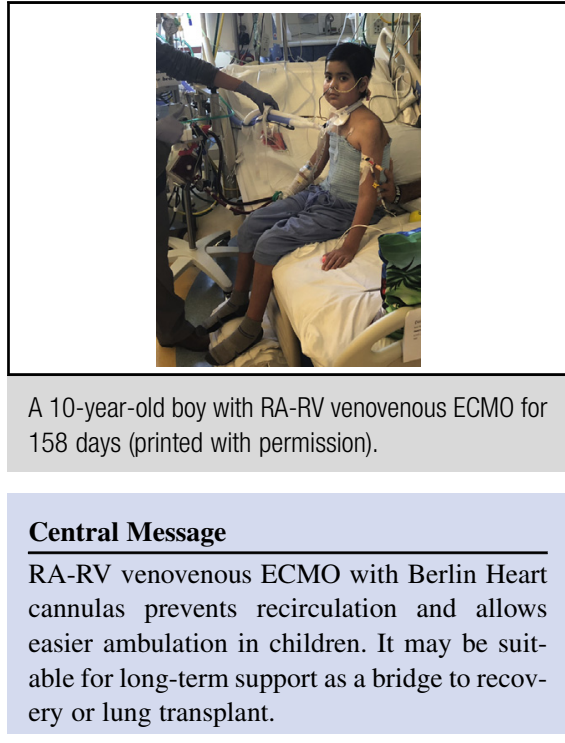

See Editorial Commentary page e15.
Venovenous extracorporeal membrane oxygenation (ECMO) has been used widely for acute respiratory failure as a bridge to recovery or to a lung transplant. ${ }^{1}$ Long-term support is often necessary, and keeping patients awake and ambulatory is critical for lung recovery and successful lung transplantation. ${ }^{2}$

In adults, a percutaneous dual-stage double-lumen venovenous cannula is commonly used. In children, however, ambulation and long-term support can be difficult with this approach because of size limitations of the cannulas, problems with recirculation, and lack of patient cooperation.

We report 2 cases of children who underwent a new venovenous ECMO cannulation strategy after failed management with a dual-stage double-lumen neck venovenous cannula. The approach comprises of a right atrial (RA) inflow cannula and a right ventricular (RV) outflow cannula, both of which are Berlin Heart cannulas (Berlin Heart $\mathrm{GmbH}$, Woodlands, Tex). This novel configuration prevents recirculation and allows easier ambulation.

\section{CLINICAL SUMMARY \\ Case 1}

A previously healthy, 5-year-old, 28.6-kg girl was seen with necrotizing pneumonia with severe bilateral lung consolidation and hemothorax. She initially underwent right neck venovenous ECMO cannulation with a $19 \mathrm{~F}$ Avalon cannula (Avalon Laboratories, LLC, Rancho Dominguez, Calif) for 13 days. Because of difficulty in lowering sedation, evidence of recirculation, and recurrent episodes of hemothorax, however, she underwent conversion to central RA-RV venovenous ECMO cannulation under general anesthesia. Through a median sternotomy, a 9-mm Berlin
Heart atrial inflow cannula was inserted into the RA, and a 6-mm Berlin Heart apical ventricular outflow cannula was inserted into the diaphragmatic surface of the RV (Figure 1). The cannulas were tunneled out to the upper abdomen and connected to the ECMO pump. The patient required ECMO for 74 more days and was able to ambulate for rehabilitation during that period. Eventually, she regained sufficient pulmonary function to allow decannulation. The RV cannula site was closed directly, and the RA cannula site was patched closed with pericardium. The patient was discharged to a rehabilitation facility 17 days later.

\section{Case 2}

A 10-year-old, $24.4 \mathrm{~kg}$ boy was seen with idiopathic fibrosing pneumonitis. He initially underwent right neck venovenous ECMO with a 27F Avalon cannula as a bridge to recovery or to a lung transplant. Complications with recirculation and high sedation needs made ambulation impossible, so after 42 days, he underwent central RA-RV cannulation for venovenous ECMO with the same technique used in case 1 . After 116 days of rehabilitation accomplished with awake, ambulatory ECMO, the patient 


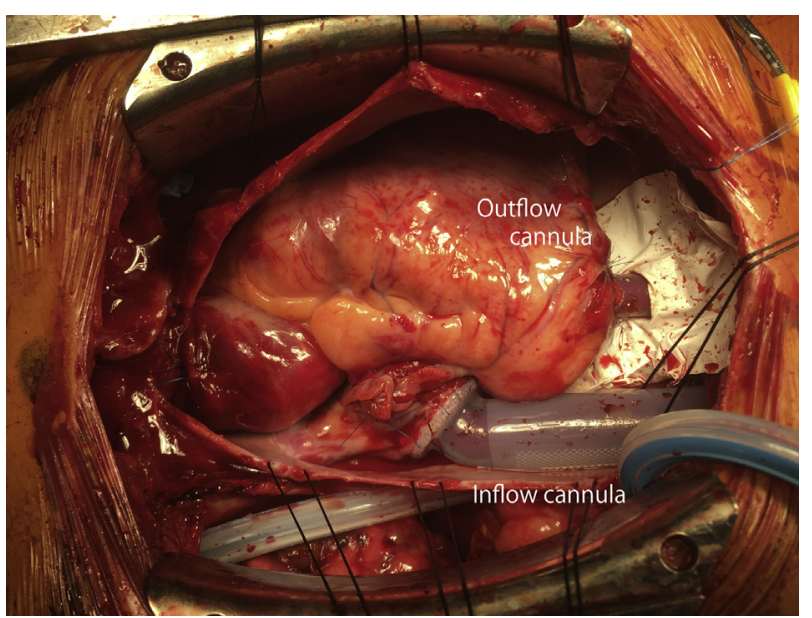

FIGURE 1. A 9-mm Berlin Heart (Berlin Heart GmbH, Woodlands, Tex) atrial inflow cannula is inserted on the right atrium, and a 6-mm Berlin Heart apical ventricular outflow cannula is placed on the diaphragm surface of the right ventricle. The expanded polytetrafluoroethylene membrane is placed around the outflow cannula to prevent adhesion.

was decannulated. He was discharged to a rehabilitation facility 22 days later.

\section{DISCUSSION}

There are 3 advantages to this technique. First, this mode prevents recirculation, because the tricuspid valve separates the inflow and outflow cannulas. Recirculation is common with dual-stage venovenous cannulas, especially in small children, and can result in inadequate support and failure to rest the lungs. Second, the cannulas exit the abdomen, allowing patients to move their heads freely and ambulate more easily. Both of these children required concomitant tracheostomy, and the risk of infecting the neck cannulas through the tracheostomy site would have been high. Berlin Heart cannulas are designed for long-term use, and the smooth silicone inner surface covered by Dacron velour (C.R. Bard, Haverhill, Pennsylvania) at the contact site at the abdominal wall encourages scar tissue ingrowth and thereby minimizes infection. ${ }^{3}$ Finally, unlike the use of an
RV assist device with an artificial membrane, this approach allows the RV to function normally and does not result in deconditioning, because volume is not off-loaded. ${ }^{4}$ In the former technique, the inflow cannula is on the RA, and the outflow cannula is in the pulmonary artery, therefore bypassing the $\mathrm{RV}$. ${ }^{4}$ The long-term effects on the function of the $\mathrm{RV}$ of using an RV assist device are not known. In the immediate postoperative period after a lung transplant, the potentially deconditioned RV may progress to failure.

There are 2 potential disadvantages to our technique. This approach requires a median sternotomy and cardiopulmonary bypass, and it should therefore be used only when percutaneous venovenous ECMO management has failed. Furthermore, the placement of the cannulas may damage the tricuspid valve or RV. Although RV function was maintained during and after ECMO in both cases, we observed mild tricuspid regurgitation after decannulation of each patient.

This novel use of Berlin Heart cannulas for central cannulation of the RA and RV for venovenous ECMO is an alternative for children who do not tolerate dual-stage venovenous cannulas. This procedure can be performed safely and prevents recirculation. It may be appropriate for longterm ambulatory ECMO support as a bridge to recovery or to a lung transplant.

\section{References}

1. Finney SJ. Extracorporeal support for patients with acute respiratory distress syndrome. Eur Respir Rev. 2014;23:379-89.

2. Biscotti M, Gannon WD, Agerstrand C, Abrams D, Sonett J, Brodie D, et al. Awake extracorporeal membrane oxygenation as bridge to lung transplantation: a 9-year experience. Ann Thorac Surg. 2017;104:412-9.

3. Hetzer R, Potapov EV, Stiller B, Weng Y, Hübler M, Lemmer J, et al. Improvement in survival after mechanical circulatory support with pneumatic ventricular assist devices in pediatric patients. Ann Thorac Surg. 2006;82: 917-24; discussion 924-5.

4. Song TH, Baker JN, Melnitchouk SI, Sundt TM, Garcia JP. Ambulatory extracorporeal membrane oxygenation with right atrium to pulmonary artery cannulation through hemisternotomy for long-term support. J Heart Lung Transplant. 2014; 33(4 Suppl):S249.

5. Banga A, Batchelor E, Mohanka M, Bollineni S, Kaza V, Mullins J, et al. Predictors of outcome among patients on extracorporeal membrane oxygenation as a bridge to lung transplantation. Clin Transplant. 2017;31(7). 